\title{
The use of the cerebroplacental ratio in the latent phase of labour to predict adverse outcomes in a resource-limited setting
}

\author{
A J Wise, ${ }^{1,2}$ MB BCh, FCOG (SA), MMed (O\&G), Dip HIV Man (SA), ACHM (FPD), Cert Maternal Fetal Med (SA); \\ E Nicolaou, ${ }^{3,4,5}$ MD, FCOG (SA), Dip Fet Med (UK); H Lombaard, ${ }^{1,2}$ MB ChB, FCOG (SA), MMed (O\&G), PG Dip HSE
}

\author{
${ }^{1}$ Department of Obstetrics and Gynaecology, Rahima Moosa Mother and Child Hospital, Faculty of Health Sciences, University of the \\ Witwatersrand, Johannesburg, South Africa \\ ${ }^{2}$ Empilweni Services Research Unit, Rahima Moosa Mother and Child Hospital, University of the Witwatersrand, Johannesburg, South Africa \\ ${ }^{3}$ Department of Obstetrics and Gynaecology, Chris Hani Baragwanath Academic Hospital; University of the Witwatersrand, Johannesburg, \\ South Africa \\ ${ }^{4}$ Morningside Mediclinic, University of the Witwatersrand, Johannesburg, South Africa \\ ${ }^{5}$ Department of Obstetrics and Gynaecology, Pavilion for Women, Texas Children's Hospital, Baylor College of Medicine, Houston, USA
}

Corresponding author: A J Wise (amyjulietwise@yahoo.co.uk)

Background. Risk stratification of pregnant women may decrease the perinatal mortality rate in South Africa. This relies on the assessment of the referring health facility and timeous presentation of the patient. Inaccurate dating affects the ability to detect growth restriction. Patients requiring referral may go undetected and deliver with the inappropriate level of care. Doppler studies can assist in detecting at-risk fetuses.

Objectives. To describe the short-term outcomes of fetuses with a normal or abnormal cerebroplacental ratio (CPR) according to the various definitions in the literature.

Methods. A cohort of 200 pregnant women were prospectively recruited while in early labour. Patient history including previous ultrasound scans were obtained. Ultrasound was done for biometry, umbilical artery (UA), middle cerebral artery (MCA) and uterine Dopplers, and the CPR was calculated. Labour and delivery details were recorded. An association between an abnormal CPR, adverse outcomes and composite score was determined.

Results. Less than a tenth $(7.7 \% ; n=15)$ of the participants had a CPR $<1.08$. Furthermore, $16.5 \%(n=31)$ of the participants were $<5$ th centile and $24.2 \%(n=47)$ were $<10$ th centile. The composite score of adverse outcomes in those with and without a CPR $<5^{\text {th }}$ centile was not significant $(p=0.737)$. There was no association between adverse outcomes $(p=0.179)$ or a composite score $(p=0.237)$ and the CPR cut-off of the 10th centile. Moreover, there was no association between an abnormal CPR and adverse outcomes after adjusting for confounders. There were no cases of perinatal death or neonatal encephalopathy.

Conclusion. An abnormal CPR was not clinically useful in detecting fetuses at risk of adverse outcomes in early labour regardless of the CPR cut-off. However, this is reassuring when normal and may if combined with other parameters still prove useful.

S Afr J Obstet Gynaecol 2020;26(2):48-53. https://doi.org/10.7196/SAJOG.2020.v26i2.1590

The role of the cerebroplacental ratio (CPR) needs to be more precisely defined as a screening tool for adverse perinatal outcomes and how it could influence management of pregnant women. ${ }^{[1,2]}$ An abnormal CPR is associated with the diagnosis of fetal compromise, caesarean section (CS), meconium-stained liquor and an abnormal cardiotocograph (CTG) in appropriate-for-gestational age (AGA) fetuses in the latent phase of labour. ${ }^{[3]}$ It is also part of the multi-vessel Doppler assessment in fetuses at risk of growth restriction. ${ }^{[4]}$

The decision as to which patients will require more intensive monitoring during labour in a resource-limited setting is complex. In South Africa (SA), fetal heart monitoring with a hand-held Doppler device for low-risk women in labour in a community clinic is recommended. In a hospital, CTG machines are used for high-risk patients. Appropriate stratification relies on the assessment by the referring health facility and timeous patient presentation. ${ }^{[5]}$

Gestational age (GA) at booking and antenatal attendance confounds attempts to accurately assess women and has implications for the detection of at-risk pregnancies. The majority of pregnant women (92.9\%) visit the antenatal clinic (ANC) at least once, but only $51.8 \%$ of women present before 20 weeks in SA. ${ }^{[6]}$ The Gauteng Province had the lowest ANC attendance of $89.9 \%$ in 2016. ${ }^{[7]}$ This results in some women who require a higher level of care going undetected.

Unexplained stillbirth is the biggest contributor to the perinatal mortality rate (PNMR). Some stillbirths are due to undiagnosed intrauterine growth restriction (IUGR). ${ }^{[8]}$ The availability of ultrasound and skilled sonographers is limited in clinics and district hospitals, making the diagnosis of IUGR difficult. A Cochrane review found insufficient evidence to conclude that using the symphysis fundal measurement results in effective detection of IUGR. ${ }^{[9]}$

More options are needed to identify at-risk fetuses in resourcelimited settings where perinatal morbidity and mortality rates are unacceptably high. We aimed to determine the percentage of fetuses with an abnormal CPR according to the various definitions, as well as the short-term fetal outcomes and their relationship with either a normal or abnormal CPR in a heterogenous population in early 
labour. If patients are identified early in labour as being at a higher risk for adverse outcomes, the allocation of resources for monitoring could be adjusted and the risk of a poor outcome may be reduced.

\section{Methods}

This was a prospective observational cohort study that recruited 200 patients from November 2017 until March 2018. Patients older than 18 years, able to understand and sign consent in English, in the latent phase of a spontaneous labour $(<4 \mathrm{~cm}$ dilatation) as diagnosed by the attending healthcare worker, and with a normal CTG as judged by the researchers according to local protocol, were included in the study ${ }^{[10]}$ Patients were excluded if the fetus was known to be growth-restricted, have a two-vessel cord, a major anomaly, multiple pregnancy and meconium-stained liquor or a previous CS.

The study was conducted in a regional academic hospital in Johannesburg, SA. The hospital serves a low-to-middle-income area with a diverse population. In 2017, 13072 babies were delivered at the hospital and a further 405 were born before arrival. The teenage pregnancy rate was $2.2 \%$ and advanced maternal age rate was 9.5\%. The CS rate was 38.2\%, PNMR was 33.4/1 000 births and the stillbirth rate was 21/1 000 births. $^{[1]]}$ There are several ANCs in the area but only two have delivery facilities. Hence, a large number of purportedly low-risk cases which ordinarily would be managed by a midwife are delivered in hospital.

There is a dedicated ultrasound machine that is available at all times in the labour ward admission area where recruitment took place. We used the SonoScape S12 digital colour Doppler ultrasound system (SonoScape, China). CTG machines are available but not in the numbers needed to ensure that each patient can be monitored continuously. Hand-held devices are not always functional and midwife staffing shortages prevent one-on-one monitoring of patients in the active phase of labour.

Ultrasounds were done by the investigators. Patient files were screened in the admission area and patients in the latent phase of labour were approached to participate in the study. If one of the exclusion criteria was detected during the ultrasound, the patient was excluded from the study.

Pregnancy was dated using the early ultrasound results, followed by the last normal menstrual period (LNMP) or the late ultrasound and the symphysis fundal height (SFH). The number of fetuses, fetal heart activity, lie, presentation, placental localisation and whether the fetus was intrauterine, as well as the number of cord vessels, were confirmed. The single deepest pool (SDP) was measured. ${ }^{[12]}$ Estimated fetal weight (EFW) was calculated using biparietal diameter, head circumference, abdominal circumference (AC) and femur length using Hadlock's formula. ${ }^{[13]}$ Uterine artery, umbilical artery (UA) and middle cerebral arteries (MCA), resistance index and pulsatility index (PI) were measured while keeping the angle of insonation as close as possible to $0^{\circ}$. Doppler measurements were performed following the International Society of Ultrasound in Obstetrics and Gynaecology (ISUOG) Doppler guidelines. CPR was calculated using the Fetal Medicine Barcelona calculator (http://medicinafetalbarcelona.org/ calc/.), which calculates the ratio and the centile and then reports it as normal or pathological. If the CPR was $<1.08$ and/or the centile $<5$ th, it was considered abnormal. ${ }^{[14,15]}$

The standard of care is to transfer patients to the labour ward in the active phase of labour (cervical dilatation $>4 \mathrm{~cm}$ ). Since an abnormal CPR is associated with adverse outcomes, it is prudent to monitor such patients closely when identified. It is not standard practice to immediately offer a CS if the CPR is abnormal. The study was not blinded and recruitment was performed by two people. This assists with consistency; however, it may also result in bias. Ethically, since there is a described association between abnormal Doppler measurements and poor outcomes, it was decided that the patients would be informed of the findings and a request made for continuous monitoring. If no bed was immediately available, continuous fetal monitoring for those with abnormal Doppler measurements was ordered in the admission ward. On-call staff then had to make a decision regarding which patients had priority for access to available resources and manage all further care of the patient as per standard labour and delivery protocols.

At the time of delivery, staff performing the delivery were requested to send an arterial cord blood gas for analysis. While regular reminders were given to staff on the procedure, it is possible that some samples may have been a mix of arterial and venous blood, which would potentially have increased the $\mathrm{pH}$ slightly - normal arterial $\mathrm{pH}$ (standard deviation (SD)) is $7.24(0.07)$ and venous $\mathrm{pH}$ is $7.33(0.06){ }^{[16]}$ The patients were informed that only cord blood would be taken for the study. Any other procedures required were part of routine care. These cord blood gas results, delivery details and short-term outcomes of the mother and neonate were collected after discharge.

Since this is a descriptive study and no prior study has been done on a heterogenous population such as ours, no power calculation was done. The decision to recruit 200 patients was based on the availability of the researchers and that at least $10 \%$ of the patients were expected to have an abnormal CPR based on the work of Prior et al. ${ }^{[3]}$ which assessed low-risk women.

As part of the analysis, both the score used by Prior et al..$^{[3]}$ and each adverse outcome was compared in patients with a CPR $>1.08$ and in those with a CPR $<1.08$ and/or $<5$ th centile. A score was worked out for all participants who had all variables available including the blood gas (Table 1).

Since not all our patients had a gas analysis performed on cord blood, a number of adverse outcome variables were considered to allow for comparison. ${ }^{[17]}$ These adverse outcome variables included a diagnosis of fetal compromise, arterial cord blood $\mathrm{pH}<7.20$, Apgar $<7$ at 5 minutes, need for resuscitation/assistance with breathing, admission to the neonatal unit and a birthweight $<10$ th centile. ${ }^{[17]}$ The Intergrowth 21 growth charts were used to determine birth centiles, the variables entered were the most accurate gestational age (calculated prior to the research ultrasound), neonatal sex and birthweight. ${ }^{[18]}$

Study data were collected using REDCap (Research Electronic Data Capture), an online database manager hosted at the University of the Witwatersrand. ${ }^{[19]}$ Descriptive statistics were used for categorical and continuous data. This was done to determine the prevalence of the variables as well as means and medians where appropriate. Certain continuous variables were categorised for descriptive and analytical purposes. Analytical statistics were

Table 1. Prior's adverse outcome score

\begin{tabular}{lllll}
\hline & $\mathbf{0}$ & $\mathbf{1}$ & $\mathbf{2}$ & $\mathbf{3}$ \\
\hline $\mathrm{pH}$ & $\geq 7.2$ & $7.1-<7.2$ & $7.0-<7.1$ & $<7.0$ \\
Base excess & $<-8$ & $\geq-8$ and $<-12$ & $\geq-12$ & \\
Apgar & $\geq 7$ at $1 \mathrm{~min}$ & $<7$ at $1 \mathrm{~min}$ & $<7$ at $5 \mathrm{~min}$ & \\
$\begin{array}{l}\text { Neonatal unit } \\
\text { admission }\end{array}$ & No & Yes & - & - \\
& & & &
\end{tabular}


employed to test for any association between the ultrasound findings and fetal outcome. Parametric testing was done with $\chi^{2}$ tests and Fisher's exact tests. A p-value of 0.05 was used to include or exclude the potential of any outcome. Where variables were missing, the denominator was adjusted. The data were analysed with the assistance of a biostatistician. Statistical analysis was performed using Statistica software, version 13.3 (Tibco Software Inc., USA)and R package, version 3.5.1 (RStudio, USA).

Ethics clearance was granted by the University of Witwatersrand Human Research Ethics Committee (ref. no. M170637). The study was registered with the National Health Research Database (ref. no. GP_201709_014). Permission to conduct the study was granted by Rahima Moosa Mother and Child Hospital.

\section{Results}

We prospectively recruited 200 patients who consented to participate in the study. We excluded two patients who had undiagnosed twins at the time of the ultrasound and six files went missing. Therefore, we had $96 \%(N=192)$ of the patients' files available for analysis and $70.5 \%(n=141)$ of the patients had cord blood gas analysis data.

The minimum age of consent is 18 years; therefore, no comment can be made about the teenage pregnancy rate. More than a tenth of the women $(13.3 \% ; n=26)$ were 35 years and older. More than onethird $(43.4 \% ; n=85)$ of the patients were primiparous. The majority (97.4\%) initiated antenatal care at a local clinic. Furthermore, the majority of the women $(73.2 \% ; n=142)$ booked before 24 weeks gestation and $30.4 \%(n=59)$ were $<14$ weeks pregnant. A third $(32.4 \%)$ were obese (body mass index (BMI) $\geq 30 \mathrm{~kg} / \mathrm{m}^{2}$ ) at their first visit. Of the women, $31.7 \%(n=45)$ had mean arterial pressure (MAP) that was $>90 \mathrm{mmHg}$ before 24 weeks. MAP was calculated from blood pressure noted in the file and was not done in a standardised manner. The mean haemoglobin $(\mathrm{Hb})$ of the cohort improved between booking and the time of delivery. At booking, $21.2 \%(n=40)$ of the patients were anaemic $(\mathrm{Hb}<11 \mathrm{~g} / \mathrm{dL})$ while at the last evaluation, $22.9 \%(n=44)$ of the patients were anaemic. Medical conditions (excluding HIV) were found in $6.7 \%(n=13)$ of the patients: 5 had asthma, 4 had hypertension and there was 1 case each of tuberculosis, neurofibromatosis, psychosis and chicken pox during pregnancy.-

Blood tests done at booking were Rhesus ( $\mathrm{Rh}$ ) status, $\mathrm{Hb}$, syphilis and HIV serology. Only $2.1 \%(n=4)$ of the patients were Rh-negative and only one was tested for antibodies, which was also negative. All patients tested negative for syphilis. The prevalence of HIV was $18.0 \%$, with a median (range) CD4 T cell count of 414 (51 - 815) cells/ $\mu \mathrm{L}$ and a HIV viral load of $<1000$ copies $/ \mathrm{mL}$ in $77.1 \%(n=27)$ of the
HIV-positive women. More than a tenth $(14.3 \% ; n=5)$ of the patients qualified for prophylaxis for Pneumocystis jirovecii pneumonia (CD4 T cells $<200$ cells $/ \mu \mathrm{L}$ ), of which two received the prophylaxis and it was not prescribed for the other three. Sixteen (45.7\%) HIV-positive patients were screened for hepatitis B and $2.9 \%(n=1)$ tested positive.

The self-reported race of each patient was as follows: $83.2 \%$ $(n=163 / 196)$ black, $12.8 \%(n=25 / 196)$ coloured, $2.0 \%(n=4 / 196)$ Asian and 2.0\% ( $n=4 / 196)$ white (Table 2$)$. The coloured and white patients were all South African. One Asian patient came from Pakistan. Of the black patients, 53.6\% $(n=105 / 196)$ were from SA, 19.4\%. ( $n=38 / 196)$ were from Zimbabwe, $4.1 \%(n=8 / 196)$ were from Malawi, $2.0 \%(n=4 / 196)$ were from Lesotho and $1 \%(n=2 / 196)$ each were from Mozambique, Kenya and Democratic Republic of Congo and $0.5 \%(n=1)$ was from Nigeria, Ethiopia and Republic of Congo, respectively. The percentage of non-South Africans was $30.1 \%(n=59)$. This is lower than our hospital average as some non-South Africans were excluded before recruitment as they were non-English speaking.

There was a significant association between gravidity and BMI of the patient with the mode of delivery (Table 3). A higher gravidity and lower BMI were associated with normal delivery (Table 3). There was no association between mode of delivery and the other variables (Table 3).

More than half of the patients $(53.1 \% ; n=103 / 194)$ had a previous ultrasound at a median gestation of 24 weeks (Table 4). The

Table 3. Mode of delivery

\begin{tabular}{|c|c|c|c|}
\hline & $\begin{array}{l}\text { Any vaginal } \\
\text { delivery }(n=134)\end{array}$ & $\begin{array}{l}\text { Caesarean } \\
\text { section }(n=58)\end{array}$ & \\
\hline & ${ }^{*} n(\%)$ & ${ }^{*} n(\%)$ & $p$-value \\
\hline Age (years), median (IQR) & $28(23-32)$ & $26(22-31)$ & 0.276 \\
\hline Race & & & 0.504 \\
\hline Black & $109(81.34)$ & $52(89.66)$ & - \\
\hline Coloured & $18(13.43)$ & $5(8.62)$ & - \\
\hline Asian & $4(2.99)$ & $0(0.0)$ & - \\
\hline White & $3(2.24)$ & $1(1.72)$ & - \\
\hline Parity, median (IQR) & $1(0-2)$ & $0(0-1)$ & 0.051 \\
\hline Gravidity, median (IQR) & $2(1-3)$ & $1(1-2)$ & 0.021 \\
\hline BMI $\left(\mathrm{kg} / \mathrm{m}^{2}\right)$, mean $(\mathrm{SD})$ & $26.95(5.91)$ & $28.89(6.01)$ & 0.047 \\
\hline $\begin{array}{l}\text { MAP at admission } \\
(\mathrm{mmHg}), \text { mean }(\mathrm{SD})\end{array}$ & $91.72(8.19)$ & $90.85(6.93)$ & 0.453 \\
\hline $\begin{array}{l}\mathrm{Hb} \text { at delivery }(\mathrm{g} / \mathrm{dL}), \\
\text { mean }(\mathrm{SD})\end{array}$ & $11.93(1.64)$ & $12.29(1.46)$ & 0.136 \\
\hline $\begin{array}{l}\text { IQR }=\text { interquartile range, } \mathrm{BMI}=\mathrm{b} \\
\mathrm{MAP}=\text { mana arterial pressure; } \mathrm{Hb} \\
\text { *Unless otherwise specified. }\end{array}$ & $\begin{array}{l}\text { ody mass index; } \mathrm{SD}=\text { sta } \\
=\text { haemoglobin. }\end{array}$ & ndard deviation; & \\
\hline
\end{tabular}

Table 2. Demographics and biophysical data

\begin{tabular}{|c|c|c|c|c|c|}
\hline & $N$ & Mean (SD) & Median & Minimum & Maximum \\
\hline Age (years) & 196 & $27.3(5.52)$ & 27.0 & 18.0 & 41.0 \\
\hline Parity & 196 & - & 0 & 0 & 4 \\
\hline Height (m) & 182 & $159.0(7.35)$ & 159.0 & 132.0 & 178.0 \\
\hline Weight (kg) & 193 & $69.7(15.21)$ & 66.0 & 44.0 & 124.0 \\
\hline BMI $\left(\mathrm{kg} / \mathrm{m}^{2}\right)$ & 182 & $27.7(6.10)$ & 26.3 & 18.1 & 55.6 \\
\hline $\mathrm{Hb}$ at booking (g/dL) & 189 & $12.0(1.66)$ & 12.1 & 6.1 & 16.2 \\
\hline Hb most recent $(\mathrm{g} / \mathrm{dL})$ & 192 & $12.0(1.59)$ & 12.1 & 6.7 & 16.0 \\
\hline
\end{tabular}


completeness of information was variable in these reports. One ultrasound appeared completely at odds with other findings and was thus not used to date the pregnancy. The GA at recruitment was used to calculate the CPR. The median (range) GA at recruitment was $39 w 3 d(33 w 2 d-44 w 6 d)$. Although the GA of 44w6d is unlikely, there was no other satisfactory way of dating her pregnancy.

The ultrasound data revealed that $16.5 \%(n=31)$ of 194 patients had a CPR that was $<5$ th centile and $7.7 \%(n=15)$ of these patients had a CPR $<1.08$ (Table 4 ). A CPR $<10$ th centile has been considered to be abnormal in other studies, ${ }^{[3]}$ but this is not a definition used by the Fetal Medicine Barcelona calculator. In our cohort, $24.2 \%(n=47)$ of the participants were $<10$ th centile. The UA PI was in the normal range for gestational age as per the Fetal Medicine Barcelona calculator for all patients (Table 4).

There were no cases of perinatal death or neonatal encephalopathy. Since there was no long-term follow-up, no comment can be made on neurodevelopmental outcomes.

A score out of eight based on Prior et al.' $\mathrm{s}^{[3]}$ work was calculated for $69.1 \%(n=134)$ of the patients who had all variables available (Table 5).

We found no association between CPR $<5$ th centile and fetal compromise diagnosed at any time during labour $(p=0.365)$, an Apgar score $<7$ at 5 minutes $(p=0.302)$, cord arterial $\mathrm{pH}<7.20$ $(p=0.104)$, small-for-gestational age (SGA) $(p=0.252)$, neonatal unit admission $(p=0.741)$, bag mask ventilation (BMV) usage/ respiratory support $(p=0.399)$ (Table 6). Prior's composite score was not significant $(p=0.737)$. When the CPR cut-off was the 10 th centile, there was again no association between adverse outcomes and any of the other variables $(p=0.179)$ or Prior's composite score $(p=0.237)$. Moreover, we still found no association between abnormal CPR and adverse outcomes even after adjusting for confounders such as age, race, parity, gravidity, BMI, hemoglobin, blood pressure, growth restriction, mode of delivery, GA, sex of the fetus and use of an epidural.

The CPR is reassuring when normal but it is a poor predictor of adverse outcomes when abnormal, except for bag mask ventilation/ resuscitation where it had a positive predictive value (PPV) of 0.70 (Table 6).

\section{Discussion}

There is a need to identify modalities that enable the detection of high-risk pregnancies in a resource-limited setting. This is challenging when despite a third (30.4\%) of women booking early enough for first trimester screening and two-thirds being eligible for a second trimester anatomy scan, ${ }^{[6]}$ only $53.1 \%$ received an ultrasound examination and these were performed by practitioners with varying levels of skill. A study by Geerts et al. ${ }^{[20]}$ reported similar findings as our study that $61.3 \%$ of their patients booked before 24 weeks; however, they reported that up to $88.9 \%$ of their patients had at least one ultrasound.

The population we serve is heterogenous with a number of nationalities (41.4\% of deliveries in 2017) $)^{[11]}$ and racial groups that differ in proportion to those in the literature, which comments on ethnicity but not on immigration status. ${ }^{[21,22]}$ We found a HIV rate of $18.6 \%$, which is in line with $18.0 \%$ reported in the literature. ${ }^{[1]}$ Anaemia is associated with SGA; ${ }^{[23]}$ however, despite a fifth $(21.2 \%)$ of the patients being anaemic at booking, only three cases of SGA developed $(p=0.353)$.
Table 4. Ultrasound data $(N=196)$

\begin{tabular}{lllll}
\hline & Mean (SD) & Median & Minimum & Maximum \\
\hline GA w/d & - & $37 \mathrm{w} 2 \mathrm{~d}$ & $34 \mathrm{w0d}$ & $41 \mathrm{w} 4 \mathrm{~d}$ \\
EFW (g) & $3220(409.2)$ & 3196 & 2202 & 4489 \\
$\begin{array}{l}\text { Liquor - } \\
\text { Single deepest } \\
\text { pocket (cm) }\end{array}$ & $3.9(1.93)$ & 3.7 & 0.4 & 18.0 \\
LUA PI & $0.83(0.60)$ & 0.75 & 0.06 & \\
RUA PI & $0.91(0.43)$ & 0.79 & 0.29 & 2.40 \\
UA PI & $0.84(0.16)$ & 0.82 & 0.49 & 2.57 \\
MCA PI & $1.35(0.29)$ & 1.33 & 0.61 & 1.27 \\
CPR & $1.65(0.42)$ & 1.61 & 0.76 & 2.76 \\
CPR centile & 35.7 & 32.0 & IQR 10.0 - 55.25
\end{tabular}

$\mathrm{SD}=$ standard deviation; $\mathrm{GA}=$ gestational age; $\mathrm{EFW}=$ estimated fetal weight; LUA = left uterine artery; PI = pulsatility index; RUA = right uterine artery; $\mathrm{UA}=$ umbilical artery; $\mathrm{MCA}=$ middle cerebral artery; $\mathrm{CPR}=$ cerebroplacental ratio.

Table 5. Prior's score

\begin{tabular}{ll}
\hline Prior's score & $\boldsymbol{n}(\%)$ \\
\hline 0 & $87(64.9)$ \\
1 & $22(16.4)$ \\
2 & $9(6.7)$ \\
3 & $5(3.7)$ \\
4 & $6(4.5)$ \\
5 & $2(1.5)$ \\
6 & $2(1.5)$ \\
7 & $1(0.7)$ \\
8 & -
\end{tabular}

Table 6. Cerebroplacental ratio, PPV and NPV for adverse outcomes

\begin{tabular}{lll}
\hline Adverse outcome & PPV & NPV \\
\hline Fetal compromise & 0.24 & 0.68 \\
Apgar 5 min $<7$ & 0.01 & 0.97 \\
Cord $\mathrm{pH}<7$ & 0.23 & 0.93 \\
SGA & 0.04 & 0.89 \\
Admission & 0.09 & 0.90 \\
BMV/resus. & 0.70 & 0.22 \\
Any adverse outcome & 0.13 & 0.92
\end{tabular}

$\mathrm{PPV}=$ positive predictive value; $\mathrm{NPV}=$ negative predictive value; $\mathrm{SGA}=$ small-for-gestational age; $\mathrm{BMV} /$ resus. = bag mask ventilation/resuscitation.

In our study, we found that $7.7 \%(n=15)$ of the participants had a CPR $<1.08,16.5 \%(n=31)$ were $<5$ th centile and $24.2 \%(n=47)$ of the participants were $<10$ th centile. A study by Prior et al. ${ }^{[3]}$ found that $10 \%$ of the patients had an abnormal ratio when defined as $<10$ th centile. This was corroborated by a more recent study which showed that $9.6 \%$ of the patients who were low risk had a CPR $<10$ th centile..$^{[2]}$

The CPR has been shown to be predictive of poor outcomes in a variety of settings and GAs. ${ }^{[17,24,25]}$ Late-onset IUGR may have a normal UA with a decreased MCA PI, which then results in an abnormal CPR. ${ }^{[26]}$ This is of particular interest since the detection of late-onset IUGR is difficult without accurate early dating. Our sample is small with $6.8 \%(n=13)$ neonates $<10$ th centile for weight and pre-existing IUGR as an exclusion criterion. 
The cut-off value of $<1.08$ has a higher sensitivity and lower specificity than using $<5$ th centile, which has a lower sensitivity but higher specificity in detecting adverse perinatal outcomes such as hypoxic ischaemic encephalopathy, periventricular leukomalacia, intraventricular haemorrhages, necrotising enterocolitis, sepsis, bronchopulmonary dysplasia and death. ${ }^{[25]}$ However, we found no association between any adverse outcomes and the 10th centile as a cut-off $(p=0.237)$.

This may indicate that our designation of some patients as low risk is incorrect and that they should have had an elective delivery earlier rather than awaiting spontaneous labour or more intensive monitoring antepartum and intrapartum. However, in a population studied just prior to induction with a much higher burden of highrisk conditions, the CPR was not shown to perform well in the clinical setting. ${ }^{[22]}$

More recently, the CPR has been questioned as a stand-alone screening tool due to poor sensitivity and specificity. ${ }^{[22]}$ The PPV was high (0.70) for the need for BMV/respiratory support and resuscitation. CPR has a very good negative predictive value (NPV) of 0.92 and is reassuring when normal, which is in keeping with the literature..$^{[2,17]}$

Figueras et al. ${ }^{[27]}$ showed that combining CPR with uterine artery Doppler measurements and the EFW or AC $<3$ rd centile improved sensitivity to 82.8 (95\% confidence interval (CI) $75.1-88.6$ ), and the NPV is clinically relevant at 88.6 (95\% CI $83.2-92.5)$. This analysis still has to be done with our data and it will be interesting to see if an association is found. It is also possible that looking at the decline in the CPR over time may be a better predictor of fetuses at risk. ${ }^{[28]}$ However, a study by Kalafat et al. ${ }^{[29]}$ showed that a single point estimate was a good predictor. This then does suggest that we could continue to pursue the option of assessment at the time of delivery, which may be our only opportunity to identify at risk fetuses if CPR is assessed in conjunction with other parameters.

\section{Study limitations}

Limitations of our work are that the results may not be applicable in a setting where antenatal screening is more rigorous with greater access to technology. However, there are many areas in the world that are similar to ours and where there is an uneven distribution of resources and a heterogenous population with a high burden of hypertension during pregnancy. During this study, there was a concern of bias since the clinicians were not blinded to the Doppler results. There was a protocol in place to request more monitoring as there was evidence of an increased risk of poor outcome. While this may bias the results, in our setting we cannot afford to increase CS rates without good evidence that it is indicated. The use of CPR is not yet incorporated into our algorithms, yet it was considered ethically wrong not to monitor patients more intensively if possibly at an increased risk.

\section{Conclusion}

The aim of this study was to provide new insights on identifying fetuses at risk in a resource-limited environment using available technology. The CPR in early labour is reassuring when normal but there is no statistically significant association with any one particular outcome for a CPR $<5$ th centile. The attempt to improve the sensitivity by including those under the 10th centile was not successful. The use of the CPR alone does not assist sufficiently in mitigating risk in a clinical setting. Recent work has theorised that other pregnancy, labour and maternal characteristics are likely to have a greater impact on the occurrence of adverse outcomes. ${ }^{[22]}$ The recommendation then is not to use CPR for screening despite its good NPV but rather to further elucidate whether it would be useful as part of a more comprehensive risk stratification protocol in early labour. Those with an abnormal CPR should be evaluated for other risk factors at the time of the ultrasound, and the labour and delivery plan adjusted accordingly.

The study showed that the provision of a scan with Doppler measurements around the time of delivery is possible in our setting and does have the benefit of detecting previously undiagnosed conditions immediately relevant to the care of the mother and fetus.

Declaration. This study was done for degree purposes (MSc (Research)). Acknowledgements. We would like to thank Prof. E Libhaber and Dr A Izu for assistance with the statistics.

Author contributions. All authors contributed to the protocol. AW and HL collected data. AW wrote the manuscript with inputs from HL and EN. All authors approved the manuscript for publication.

Funding. None.

Conflicts of interest. None.

1. Vollgraff Heidweiller-Schreurs CA, de Boer MA, Heymans MW, et al. Prognostic accuracy of cerebroplacental ratio and middle cerebral artery Doppler for adverse perinatal outcome: Systematic review and meta-analysis. Ultrasound Obstet Gynecol 2018;51(3):313-322. https://doi Systematic review and $\mathrm{n}$
$\mathrm{org} / 10.1002 /$ uog. 18809

2. Dall'Asta A, Ghi T, Rizzo G, et al. Cerebroplacental ratio assessment in early labor in uncomplicated term pregnancy and prediction of adverse perinatal outcome: Prospective multicentre study. Ultrasound Obstet Gynecol 2019;53(4):481-487. https://doi.org/10.1002/uog.19113

3. Prior T, Mullins E, Bennett P, Kumar S. Prediction of intrapartum fetal compromise using the cerebroumbilical ratio: A prospective observational study. Am J Obstet Gynecol 2013;208(2):124 e1-6. https://doi.org/10.1016/j.ajog.2012.11.016 016

4. Figueras F, Caradeux J, Crispi F, et al. Diagnosis and surveillance of late-onset fetal growth restriction. Am J Obstet Gynecol 2018;218(2):S790-S802 https://doi.org/10.1016/j.ajog.2017.12.003 5. National Department of Health (NDoH). Guidelines for Maternity Care in South Africa. A Manual for Clinics, Community Health Centres and District Hospitals. 4th ed. Pretoria: NDoH, 2015. http://www.health.gov.za/index.php/2014-03-17-09-09-38/policies-and-guidelines/category/2302015p\# accessed 26 April 2016.

6. Statistics South Africa (StatsSA). Millennium Development Goals 5: Improve Maternal Health. Pretoria:StatsSA, 2015. http://www.statssa.gov.za/MDG/MDG_Country\%20Report_Final30Sep2015. pdf (accessed 29 March 2017).

7. National Department of Health (NDoH) SSASS, Medical Research Council (SAMRC), and ICF. South Africa Demographic Health Survey 2016: Key Indicators. Pretoria, South Africa and Rockville, Maryland, USA, 2017. https://www.statssa.gov.za/publications/Report\%2003-00-09/ Report\%2003-00-092016.pdf (accessed 26 October 2018).

8. Pattinson R, Rhoda N. Saving babies 2012 - 2013: Ninth report on perinatal care in South Africa. Pretoria: Tshepesa Press; 2014.

9. Robert PJ, Ho JJ, Valliapan J, Sivasangari S. Symphysial fundal height (SFH) measurement in pregnancy for detecting abnormal fetal growth. Cochrane Database Syst Rev 2012(7):CD008136. https://10.1002/14651858.CD008136.pub2

10. Bera E. Wits Obstetrics 2017 Protocol Book. Johannesburg: University of the Witwatersand, 2016

11. Bera E. Rahima Moosa Mother-and-Child Hospital Statistics: 2017. Summary for 01 January 31 December 2017.

12. Kehl S, Schelkle A, Thomas A, et al. Single deepest vertical pocket or amniotic fluid index as evaluation test for predicting adverse pregnancy outcome (SAFE trial): A multicentre, openlabel, randomised controlled trial. Ultrasound Obstet Gynecol 2016;47(6):674-679. https://doi. org/10.1002/uog.14924.

13. Hadlock FP, Harrist RB, Martinez-Poyer J. In utero analysis of fetal growth: A sonographic weight standard. Radiology 1991;181(1):129-133. https://doi.org/10.1148/radiology.181.1.1887021

14. Gramellini D, Folli MC, Raboni S, et al. Cerebral-umbilical Doppler ratio as a predictor of adverse perinatal outcome. Obstet Gynecol 1992;79(3):416-420. https://doi.org/10.1097/00006250199203000-00018

15. Baschat AA, Gembruch U. The cerebroplacental Doppler ratio revisited. Ultrasound Obstet Gynecol 2003;21(2):124-127.https://doi.org/10.1002/uog.20

16. Victory R, Penava D, da Silva $\mathrm{O}$, et al. Umbilical cord $\mathrm{pH}$ and base excess values in relation to adverse outcome events for infants delivering at term. Am J Obstet Gynecol 2004;191(6):20212028. https://doi.org/10.1016/j.ajog.2004.04.026

17. Conde-Agudelo A, Villar J, Kennedy SH, Papageorghiou AT. Predictive accuracy of cerebroplacental ratio for adverse perinatal and neurodevelopmental outcomes in suspected fetal growth restriction: ratio for adverse perinatal and neurodevelopmental outcomes in suspected fetal growth restriction:
Systematic review and meta-analysis. Ultrasound Obstet Gynecol 2018;52(4):430-441. https://doi. org/10.1002/uog.19117

18. Villar J, Cheikh Ismail L, Victora CG, et al. International standards for newborn weight, length, and head circumference by gestational age and sex: The newborn cross-sectional study of the INTERGROWTH-21st Project. Lancet 2014;384(9946):857-868. https://doi.org/10.1016/S01406736(14)60932-6

19. Harris PA, Thielke R, Payne J, et al. Research electronic data capture (REDCap) - a metadata- 


\section{CASE REPORT}

driven methodology and workflow process for providing translational research informatics support. J Biomed Inform 2009;42(2):377-381. https://doi.org/10.1016/j.jbi.2008.08.010

20. Geerts L, Poggenpoel E, Theron G. A comparison of pregnancy dating methods commonly used in South Africa: A prospective study. S Afr Med J 2013;103(8):552-556. https://doi.org/10.7196/ samj. 6751

21. Miranda J, Triunfo S, Rodriguez-Lopez M, et al. Performance of third-trimester combined screening model for prediction of adverse perinatal outcome. Ultrasound Obstet Gynecol 2017;50(3):353-360. https://doi.org/10.1002/uog.17317

22. Fiolna M, Kostiv V, Anthoulakis C, et al. Prediction of adverse perinatal outcome by cerebroplacenta ratio in women undergoing induction of labor. Ultrasound Obstet Gynecol 2019;53(4):473-480. https://doi.org/10.1002/uog.20173

23. Ota E, Ganchimeg T, Morisaki N, et al. Risk factors and adverse perinatal outcomes among term and preterm infants born small-for-gestational-age: Secondary analyses of the WHO multi-country survey on maternal and newborn health. PLoS ONE 2014;9(8):e105155. https://doi.org/10.1371/ jurvey on maternal

24. Bahado-Singh RO, Kovanci E, Jeffres A, et al. The Doppler cerebroplacental ratio and perinatal outcome in intrauterine growth restriction. Am J Obstet Gynecol 1999;180(3 Pt 1):750-756. https:// doi.org/10.1016/s0002-9378(99)70283-8
25. DeVore GR. The importance of the cerebroplacental ratio in the evaluation of fetal well-being in SGA and AGA fetuses. Am J Obstet Gynecol 2015;213(1):5-15. https://doi.org/10.1016/j. ajog.2015.05.024

26. Oros D, Figueras F, Cruz-Martinez R, et al. Longitudinal changes in uterine, umbilical and fetal cerebral Doppler indices in late-onset small-for-gestational age fetuses. Ultrasound Obstet Gyneco 2011;37(2):191-195. https://doi.org/10.1002/uog.7738

27. Figueras F, Savchev S, Triunfo S, et al. An integrated model with classification criteria to predict small-for-gestational-age fetuses at risk of adverse perinatal outcome. Ultrasound Obstet Gynecol 2015;45(3):279-285. https://doi.org/10.1002/uog.14714

28. Khalil A, Thilaganathan B. Role of uteroplacental and fetal Doppler in identifying fetal growth restriction at term. Best Pract Res Clin Obstet Gynaecol 2017;38:38-47. https://doi.org/10.1016/j. bpobgyn.2016.09.003

29. Kalafat E, Ozturk E, Sivanathan J, et al. Longitudinal change in the cerebroplacental ratio and the risk of stillbirth. Ultrasound Obstet Gynecol 2019;54(4):492-499. https://doi.org/10.1002/uog.20193

Accepted 6 November 2020. 